\title{
INTEGRACIÓN REGIONAL Y CONVERGENCIA EN CONTEXTO INTERNACIONAL ADVERSO
}

\author{
REGIONAL INTEGRATION AND CONVERGENCE IN AN ADVERSE \\ INTERNATIONAL CONTEXT
}

Alan Fairlie Reinoso *

\section{RESUMEN}

El escenario internacional favorable cambió en los últimos años, se complicó con cambios políticos en EEUU, la guerra comercial con China y se agudizó con la pandemia. Esto dificulta el éxito de tradicionales políticas de exportación de materias primas. El artículo plantea la necesidad de retomar la integración regional y los procesos de convergencia que se venían dando en Sudamérica, que pueden tener un rol crucial en el nuevo entorno internacional.

Palabras clave: integración regional, convergencia sudamericana, guerra comercial, pandemia.

\section{SUMMARY}

In recent years, the favorable international scenario has changed, it has complicated with political changes in the United States, the trade war with China and was exacerbated by the pandemic. This hinders the success of traditional raw material export policies. The article raises the need to resume regional integration and the convergence processes that were taking place in South America, which may have a crucial role in the new international environment.

Keywords: regional integration, south american convergence, trade war, pandemic.

Antes de la llegada de la pandemia mundial, las dos principales potencias mundiales se encontraban en medio de negociaciones para poner fin a una guerra tecnológica y comercial que duró aproximadamente dos años, y que habían tenido impactos negativos sobre el comercio

\footnotetext{
* Licenciado en Economía en la Pontificia Universidad Católica del Perú. Tiene un Magíster en Comercio Internacional y Desarrollo en el Instituto Torcuato Di Tella. Maestría en Política y Gestión Universitaria en la Universidad de Barcelona. Ex Decano de la facultad de Ciencias Sociales (2011-2016). Profesor Principal del Departamento Académico de la Pontificia Universidad Católica del Perú y Parlamentario Andino por la Representación del Perú periodo 2016 - 2021. Es miembro del Centro de Investigaciones Sociales, Políticas, Económicas y Antropológicas (CISEPA) de la PUCP. Director de la Maestría en Biocomercio y Desarrollo Sostenible de la PUCP.
}

Con el presente artículo, el autor formaliza su incorporación como Miembro Asociado, conforme a lo dispuesto por el Consejo Directivo de la Sociedad Peruana de Derecho Internacional, mediante Acta del 13 de diciembre de 2018. 
y el crecimiento mundiales. El cambio hacia el unilateralismo de la política comercial de EEUU, tuvo importantes implicancias.

Con la llegada de la pandemia, diversos países, entre ellos China y EE.UU., empezaron a restringir exportaciones de bienes esenciales y de equipos de protección y ventilación, que son de gran importancia en la lucha contra el Covid-19. Asimismo, EE.UU. mediante la suspensión del financiamiento a la OMS volvió a retomar medidas que afectan de manera negativa el multilateralismo y la cooperación internacional. Anteriormente, se había retirado de compromisos para enfrentar cambio climático, y de la Unesco.

Frente a este nuevo escenario internacional, con nuevos proteccionismos, acelerados cambios tecnológicos y el unilateralismo como práctica de algunos países desarrollados, los países pequeños o medianos como los de América Latina son los más perjudicados. Debido a ello, la integración regional y la convergencia sudamericana, se presentan como uno de los instrumentos para disminuir el impacto negativo de un menor dinamismo a nivel mundial y enfrentar la actual crisis internacional.

A continuación, hacemos un recuento de los acontecimientos de esta guerra comercial y tecnológica entre EE.UU. y China, y de las medidas comerciales frente a la pandemia, que configuran un preocupante escenario internacional. Asimismo, se realiza una revisión de la literatura de los avances realizados en torno a la integración regional y la convergencia sudamericana. Planteamos la necesidad de persistir en respuestas coordinadas desde los acuerdos de integración para enfrentar la emergencia, y el periodo post covid-19.

\section{Unilateralismo y Guerra comercial ${ }^{1}$}

El giro de política comercial hacia medidas proteccionistas implementadas por Estados Unidos y las disputas con China, han afectado el multilateralismo que ya venía en crisis por el estancamiento de las negociaciones en la OMC y limitación de su mecanismo de solución de controversias.

La elección de Donald Trump en noviembre de 2016 y su asunción como presidente de EE.UU. generó incertidumbre por las declaraciones realizadas durante la campaña electoral sobre el TPP, las cuales cumplió en enero de 2017. El presidente, mediante una orden del ejecutivo retiró a Estados Unidos del TPP, después de seis años de negociaciones y haber sido el principal promotor.

La nueva política comercial de Estados Unidos, priorizaría los tratados bilaterales sobre los multilaterales. Así, se observa un giro hacia el proteccionismo. Trump adujo que el pacto multilateral perjudica a los empleos estadounidenses, y los sindicatos estadounidenses presionaban argumentando que el TPP fomentaría que empleos industriales bien remunerados, sean trasladados a países donde la mano de obra es más barata.

\footnotetext{
${ }^{1}$ Basado en los artículos: "Guerra comercial entre China y Estados Unidos: entorno internacional preocupante" (http://alanfairliereinoso.pe/?p=4899), "Nueva negociación entre China y Estados Unidos" (http://alanfairliereinoso.pe/?p=5515), "Ojalá se consolide el acuerdo inicial China y EE.UU." (http://alanfairliereinoso.pe/?p=5580).
} 
La salida de EEUU también afectó a Japón, que consideró el pacto como una forma estratégicamente importante de contrarrestar el crecimiento de China en la región. ${ }^{2}$

El TPP, buscó constituir un acuerdo de integración profunda, un mega-proceso de implicancias globales. Este incluye nuevas cuestiones como coherencia regulatoria, competencia y contratación pública. El acuerdo se articula básicamente del sistema de solución de controversias. Las obligaciones del acuerdo eran de gran profundidad, instituyendo nuevos compromisos en servicios financieros, comercio electrónico, acceso de mercados, medio ambiente, trabajo, competencia, entre otros temas que lo distinguen de otros acuerdos asiáticos. De esta manera, superaba los compromisos y profundidad de acuerdos bilaterales existentes entre algunos de sus miembros (Cepal, 2015).

Sin embargo, a pesar del retiro de Estados Unidos, el resto de los miembros se enfocaron en llegar a un acuerdo para los 11 países restantes. Japón, la mayor economía entre los 11, quien asumió el liderazgo. ${ }^{3}$

En 2018, se firmó el "Tratado Integral y Progresista de Asociación Transpacífico" (CPTPP), conocido también como el TPP11. Estados Unidos en el TPP representaba el mayor valor económico. Sin EE.UU., el TPP 11 solo un equivale al 13,4\% del PBI mundial, mientras que las exportaciones e importaciones representan el $15 \%$ respectivamente del total mundial.

Según Trump, el giro de la política comercial se debe a que la apertura comercial ha puesto en una situación de desventajas a Estados Unidos, al tener un amplio déficit comercial que genera la pérdida de trabajos por la competencia extranjera.

Es así que, entre las principales acciones que ha llevado Estados Unidos, ha sido el uso de medidas antidumping como instrumento de defensa comercial (como investigaciones antidumping ( $\mathrm{AD}$ ) y de derechos compensatorios), para bloquear según criterio estadounidense las "importaciones desleales" (Kanitz, R. y Barros, M.; 2017). De otro lado, también lanzó la orden ejecutiva instando a la compra y contratación a empresas estadounidenses; ante lo cual la UE, Canadá, China y otros países cuestionaron dicha política, exhortando a Estados Unidos a cumplir con el pacto de no discriminación de la OMC y la apertura de mercado en contratación pública. ${ }^{4}$ También, renegoció el NAFTA buscando cambios en normas de origen y mayor componente de EEUU en las cadenas de valor.

Pero, el tema más complejo era la redefinición de la relación con China. La guerra comercial entre China y EE.UU. se inició en marzo de 2018, cuando el presidente de EE.UU. anunció la intención de aumentar los aranceles a las importaciones de China, argumentando prácticas desleales de comercio y robo de la propiedad intelectual. En represalia, el gobierno de China respondió anunciando un alza de aranceles a los productos provenientes de EE.UU.

\footnotetext{
${ }^{2}$ Japan Times (13/07/2017) TPP states agree new framework needed to implement pact without U.S. Recuperado de: http://www.bilaterals.org/?tpp-states-agree-new-framework\&lang=en

3 Véase Peñaver, María (12/07/2017) El TPP se reúne en Japón para discutir su futuro. Recuperado de: http://www.bilaterals.org/?el-tpp-se-reune-en-japon-para\&lang=en

${ }^{4}$ Véase Expansión (18/10/2017) Varios miembros de la OMC cuestionan la política proteccionista de Trump. Recuperado de: https://expansion.mx/economia/2017/10/18/varios-miembros-de-la-omc-cuestionan-la-politicaproteccionista-de-trump
} 
El 6 de junio de 2018, ambos países aumentan sus aranceles a las importaciones por un valor de 34,000 millones de dólares, con lo que se dio el inicio oficial de la guerra comercial. Dos meses después, se registró la segunda alza de aranceles por ambos países donde en promedio gravarían 16,000 millones de dólares cada uno. Un mes más tarde, EE.UU. vuelve a aumentar sus aranceles sobre 200,000 millones de dólares de importaciones adicionales, y China responde con un alza sobre nuevas importaciones por un valor de 60,000 millones dólares. Después de ocho meses, en mayo del 2019, EE.UU. sube los aranceles a un 25\%. Un mes más tarde, China eleva sus aranceles a una tasa similar.

Frente a estas tensiones comerciales, el 2 de junio de 2019, la Oficina de Información del Consejo de Estado de China emitió un "Libro Blanco sobre la posición de China sobre las consultas económicas y comerciales entre China y EE.UU.", donde China declara su firme posición de estar comprometida con consultas creíbles basadas en la igualdad y el beneficio mutuo y resalta que no dará lugar a cuestiones de principio destacando que ambos países deben respetar las diferencias en su desarrollo nacional y las instituciones de cada uno.

En la cumbre del G20, realizada los días 28 y 29 de junio, los presidentes de EE.UU. y China pactaron una tregua en su guerra comercial acordando restablecer las conversaciones económicas y comerciales entre los dos países. Estados Unidos accedió a no imponer nuevos aranceles sobre sus importaciones chinas y que sus empresas puedan vender productos y componentes a la tecnología china Huawei. A cambio, China accedió a comprar más bienes agrícolas estadounidenses.

Posteriormente, en la cumbre del G7, realizada del 24 al 26 de agosto, El gobierno de EE.UU. confirmó reanudar estas negociaciones para lograr un acuerdo con China. Asimismo, en la declaración oficial, los líderes del G7 manifestaron que es necesario revisar algunas de las normas de la Organización Mundial del Comercio (OMC) para hacerla más efectiva en la solución de problemas relacionados con la protección de la propiedad intelectual y de esta manera se elimine las prácticas comerciales desleales.

No obstante, una semana después, el primero de septiembre de 2019, EE.UU. volvió a subir sus aranceles por el valor de 112 mil millones de dólares. Asimismo, el presidente de EE.UU. decidió retrasar la introducción de aranceles del 10\% sobre ciertos productos chinos electrónicos por el valor de 160 mil millones de dólares -como ordenadores portátiles, videoconsolas de juegos y teléfonos móviles, entre otros- hasta el 15 de diciembre.

El 13 de diciembre de 2019, China y EE.UU. firmaron un "principio de acuerdo", en donde ambas economías a través de nueve capítulos establecen diversos compromisos con el objetivo de poner fin a la guerra comercial, siendo uno de ellos el retiro de gradual de algunos de los aranceles que han ido aplicando en los últimos 18 meses y la cancelación de EE.UU. de los aranceles programados para el 15 de diciembre.

Tras este acuerdo, Estados Unidos reduciría de $15 \%$ a $7.5 \%$ los aranceles estadounidenses, lo cual representa un valor de 120 mil millones de dólares en importaciones chinas. Por su parte, China anunció que el 1 de enero del 2020 reducirá sus aranceles a más de 850 productos extranjeros, implementado de esta forma la primera fase del acuerdo comercial con Estados

\footnotetext{
${ }^{5}$ Véase: Xinhua Español (02/06/2019) China pública libro blanco sobre su postura en consultas económicas y comerciales con EEUU. Recuperado de: http://spanish.xinhuanet.com/2019-06/02/c_138110238.htm
} 
Unidos. Además, variará sus importaciones de productos provenientes de este país especialmente los derivados de la manufactura, servicios energéticos y servicios financieros por un valor de 200 mil millones de dólares en un lapso de 2 años.

\section{Guerra tecnológica ${ }^{6}$}

El veto de Huawei, considerada como una de las firmas tecnológicas más grandes del mundo, marcó una nueva dimensión en la batalla liderada por estas dos potencias. Por un lado, Estados Unidos justificó su accionar señalando que esta medida obedece a temas de seguridad nacional, puesto que existía sospecha de espionaje a favor del gobierno de China, dadas las relaciones entre esta compañía y el gobierno chino; además, de que esta compañía ya fue vetada como contratista del Gobierno de Canadá y Australia en el 2012. Es así que, el 28 de enero del 2019, los funcionarios de seguridad nacional de Estados Unidos, acusaron a Huawei por 23 cargos criminales, entre los que se encuentran conspiración para defraudar, robo de tecnología de secreto comercial y fraude por correo electrónico.

Por su parte el Gobierno chino, anunció que continuará impulsando sus políticas y el apoyo comercial para el uso de la red 5G. Asimismo, señaló que fomentará el desarrollo de tecnología inalámbrica ultrarrápida; además, afirmó que los productos relacionados con la red 5G, entre los que se encuentran los chips y terminales, están casi listos para el uso, y que la tecnología $5 \mathrm{G}$ se utilizará en áreas clave, como Internet y sistemas industriales ${ }^{7}$.

Es así la lucha de EE.UU. y China por el futuro de la tecnología. El propio presidente de EE.UU. sostuvo en una ocasión: "Quiero la tecnología 5G, e incluso 6G, en Estados Unidos lo antes posible. Las compañías estadounidenses deben intensificar sus esfuerzos, o quedarse atrás. No hay ninguna razón por la que debamos rezagarnos en algo que obviamente es el futuro" ${ }^{\text {. }}$

Estas tensiones comerciales y políticas, afectaron el crecimiento del comercio y la economía mundial que ya tenían un deterioro. No solo menores tasas de crecimiento del comercio mundial, sino un estancamiento de las cadenas globales de valor, que se sumaban al estancamiento de las negociaciones multilaterales. La pandemia terminó de complicar el panorama y se prevé un desplome del comercio mundial, caída de precios de materias primas, del PBI mundial, turismo y servicios, remesas de migrantes, que afectan severamente a los países en desarrollo. Además de la retracción de los flujos de capital de mercados emergentes, y el creciente endeudamiento externo (BID, 2020; CEPAL, 2020; OMC, 2020; UNCTAD, 2020).

\section{Escenario se complica con la pandemia ${ }^{9}$}

Desde la rápida propagación del Covid-19, diversas economías anunciaron medidas de apoyo para mitigar su impacto económico. Unas son compatibles con el libre comercio, y otras más bien han puesto trabas al mismo. Entre las primeras, está la reducción o eliminación temporal de aranceles sobre productos médicos para reducir el potencial riesgo de escasez. Inclusive

\footnotetext{
6 Basado en el artículo "La guerra tecnológica entre China y Estados Unidos" (http://alanfairliereinoso.pe/?p=3811).

7 Véase CGTN (23/05/2019) El gobierno chino refuerza el apoyo al desarrollo de la red 5G. Recuperado de: https://www.youtube.com/watch?v=9rtMn5VTD_k

${ }^{8}$ Ibíd.

${ }^{9}$ Basado en los artículos: "Comercio de bienes esenciales en la pandemia" (http://alanfairliereinoso.pe/?p=6167),

"Unilateralismo y multilateralismo en la pandemia" (http://alanfairliereinoso.pe/?p=6290).
} 
Estados Unidos, optó por reducir sus tarifas arancelarias a productos chinos que le ayudarán a enfrentar pandemia. Todo ello iba en línea con las normas de la OMC de transparencia y no discriminación.

Sin embargo, desde mediados de marzo, los países también empezaron a restringir exportaciones de bienes esenciales. Entre los productos que han sido catalogados como de importancia en la lucha contra el Covid-19 se encuentran máscaras, guantes estériles, gafas protectoras, equipos desechables, termómetros, equipos médicos de alta tecnología, entre otros. El 15 de marzo, la Comisión Europea impuso restricciones a exportaciones de equipos de protección y ventiladores, y con el paso de los días se fueron sumando más de una docena de otras economías (incluyendo China) que en las últimas semanas restringieron o prohibieron exportaciones de estos productos. Estados Unidos a la exportación de máscaras, guantes y otros equipos de protección contra Covid-19. No son solo medidas unilaterales, sino que violan compromisos de diferentes acuerdos comerciales regionales (por ejemplo, Canadá pidió ser eximido de las medidas estadounidenses).

Por otro lado, el 14 de abril de 2020, el presidente de EE.UU. anunció la suspensión del financiamiento a la Organización Mundial de la Salud (OMS). Según su declaración, este congelamiento será hasta que se investigue el papel de la OMS en el manejo de la propagación del coronavirus. Cabe mencionar que, desde 1948 Estados Unidos ha aportado a la OMS, y que era el principal contribuyente de esta organización. Para el periodo 2018-2019, sus aportes representaron aproximadamente el 15\%, equivalente a 553 millones de dólares. Tras el congelamiento del financiamiento de Estados Unidos, China ha decidido donar 30 millones de dólares a la OMS, para la prevención y control de la pandemia y apoyar los sistemas sanitarios de los países en vías de desarrollo.

Esta situación extrema, también pone en riesgo la seguridad alimentaria, no necesariamente por la interrupción de las cadenas de suministro de manera generalizada, sino por la caída de ingresos para amplios segmentos de población, que no tendrán como acceder a los alimentos. De allí, la necesidad de adoptar medidas urgente (FAO-CEPAL, 2020b).

Si bien las respuestas de los países latinoamericanos han sido básicamente individuales, nos parece clave el rol que pueden jugar los acuerdos de integración regional no solo en la emergencia, sino en el período post-covid19.

\section{Integración regional y convergencia sudamericana}

En los últimos años, América Latina ha crecido a un ritmo muy lento. Debido a la pandemia, se estima que la región caerá en 5,2\% este año y aún existe incertidumbre de la recuperación en el 2021.

En este sentido, la integración regional se presenta como una de las vías para generar y facilitar el crecimiento de la región, además de disminuir el impacto negativo de un menor dinamismo a nivel mundial.

La ALADI en el Tratado de Montevideo (1980) define la convergencia como la multilateralización progresiva de los acuerdos de alcance parcial, mediante negociaciones periódicas entre los países miembros, en función del establecimiento del mercado común 
latinoamericano. Existen diversas propuestas de mecanismos de convergencia de la CAN, la UNASUR, el MERCOSUR y la integración sudamericana.

El BID (2018b), insta a seguir una estrategia de convergencia en la diversidad en la región, dado el contexto económico internacional y señala que la inserción internacional de los países latinoamericanos puede ser mejorada mediante un mercado regional integrado, con canastas exportadoras más diversificadas y con bienes y servicios con mayor valor agregado. EL BID ha propuesto una hoja de ruta con el objetivo de promover la convergencia entre los distintos procesos de integración regionales, reconociendo que estos poseen temas como inversiones, reglas de origen, servicios, reglas de competencia y de acceso a mercados que coinciden. Dichos puntos de intersección pueden ser la base para enlazar estos acuerdos, lo que a la vez fortalece la competitividad de las pymes al reducir los costos del comercio por la profundización de la liberalización de bienes y servicios (CEPAL, 2018b).

Desde la perspectiva del Banco Mundial (2017), diseñar nuevas políticas públicas que posibiliten el desarrollo de cadenas de valor, resulta estratégico para la integración en la región. Esto supone la articulación y complementariedad de las economías, sectores, y procesos productivos, transitar hacia una integración profunda que genere un mercado interno ampliado, la inserción de las Pymes suscitando su internacionalización y el impulso de clúster exportadores, la armonización de normas técnicas, normas de origen, y medidas de facilitación del comercio que avancen hacia un mayor comercio intrarregional.

De acuerdo con la CEPAL (2018b), el camino hacia la convergencia es la integración productiva, se precisa de paquetes integrados multinacionales de política industrial en sectores específicos, además de promover conjuntamente la innovación, transferencia tecnológica, apoyo a las pymes y la creación de clusters. En términos de la integración física, se requiere priorizar inversión en logística, transporte e infraestructura orientados el desarrollo de los clusters.

Asimismo, estudios empíricos muestran que los procesos de integración han generado una mayor convergencia de los países miembros. En un trabajo realizado por Sperlich y Sperlich (2014) se muestra que la integración Sur-Sur ha generado que la dispersión del ingreso ha disminuido ligeramente, dentro y entre los estados miembros. En la misma línea, König (2015) después de mostrar evidencia que el tamaño del país se correlaciona con el crecimiento económico para el caso europeo, señala que la integración económica europea ha mejorado el proceso de convergencia de sus países miembros. En un estudio reciente de Gnangnon (2019) se encuentra que la integración comercial tiene un efecto positivo y significativo sobre la convergencia de los países de en transición para los países de bajos, medios y altos ingresos.

Diversos espacios han analizado la posibilidad de convergencia entre los acuerdos de integración económica que coexisten en América del Sur. Es así que, en la Primera Reunión de Jefes de Estado de la Comunidad Sudamericana de Naciones, en 2005, se encargó a las Secretarías de ALADI; MERCOSUR y CAN, realizar un estudio sobre la convergencia de los acuerdos de complementación económica entre los países sudamericanos ${ }^{10}$.

\footnotetext{
${ }^{10}$ Véase: http://cosiplan.org/Event/Detail?Id=146
} 
En la Declaración de Bogotá del 8 noviembre de $2011^{11}$, el Consejo Presidencial Andino solicitó a la Secretaria General de la CAN, Secretaría General del UNASUR y a la del MERCOSUR; identificar elementos comunes y complementarios con el fin de analizar las posibilidades de convergencia de dichos procesos.

En octubre de 2014, el secretario general de la Comunidad Andina y el secretario general de UNASUR se comprometieron a trabajar conjuntamente para impulsar la convergencia de los espacios regionales de integración. Asimismo, reconocieron que la CAN, la Alianza del Pacífico, Mercosur, y el ALBA, coinciden en algunos ejes temáticos lo cual invita a "buscar espacios de convergencias para evitar duplicidades y para sumar esfuerzos" ${ }^{\prime 2}$.

En 2015, el MERCOSUR presentó una propuesta de Plan de Acción a la Alianza del Pacífico ${ }^{13}$ que busca profundizar los compromisos sobre acceso a mercado tomando como base los Acuerdos de Complementación Económica que posee con Chile, Colombia y Perú firmados en el marco de ALADI; y medidas que incidan en la facilitación de comercio intrarregional.

En el seminario "Los futuros del Mercosur. Nuevos rumbos de la integración regional" celebrado el 21 de marzo de 2017 en Buenos Aires, el BID hace un llamado a la convergencia latinoamericana y una mayor integración entre los países.

El 19 de julio de 2017, el BID llevó a cabo el seminario "MERCOSUR-Alianza del Pacífico. Una agenda positiva para la integración"14 en el marco Cumbre del MERCOSUR y Estados asociados en Mendoza, Argentina. Dicho evento se realizó por la Decisión de la Reunión Ministerial entre ambos bloques en abril de 2017. Las áreas de trabajo de dicha actividad fueron Cadenas Regionales de Valor, la Facilitación del Comercio, Cooperación Aduanera, entre otros; con el objetivo de identificar acciones pro integración.

De acuerdo a la Matriz Insumo- Producto Andina elaborada por la CEPAL (2018), los países que conforman la CAN, poseen escasos encadenamientos internos hacia atrás pero más encadenados hacia adelante. Asimismo, muestra un claro vínculo entre Colombia, Ecuador y Perú respecto a la descomposición de insumos intermedios importados según origen, además el valor agregado en bienes intermedios es alto en comparación de valor agregado doméstico en bienes finales. Con esto se concluye que es factible la integración de la base productiva en la región.

Si bien la región ha avanzado en lograr un mercado más integrado, aún persisten restricciones de acceso. El esfuerzo de convergencia es generar una plataforma para las cadenas regionales

\footnotetext{
11 Véase: Declaración de Bogotá. de: https://www.cancilleria.gov.co/sites/default/files/news/field_attached_news/Declaraci\%C3\%B3n\%20de\%20Bo got $\%$ C3\%A1\%20adoptada\%20por\%20el\%20Consejo\%20Presidencial\%20Andino\%20\%20Bogot\%C3\%A1,\%20noviembre\%208\%20de\%202011.pdf

12 Véase Secretaria General de la Comunidad Andina (22/10/2014) CAN y UNASUR trabajarán conjuntamente para promover convergencia de mecanismos regionales de integración Recuperado de: http://www.comunidadandina.org/Prensa.aspx?id=3527\&accion=detalle\&cat=AF\&title=can-y-unasurtrabajaran-conjuntamente-para-promover-convergencia-de-mecanismos-regionales-de-integracion

${ }^{13}$ Véase: Alianza del Pacífico (24 de julio de 2018) Alianza del Pacífico y Mercosur definen Plan de Acción para fortalecer vínculos entre los dos bloques. Recuperado de: https://alianzapacifico.net/alianza-del-pacifico-ymercosur-definen-plan-de-accion-para-fortalecer-vinculos-entre-los-dos-bloques/

${ }^{14}$ Véase Conexión Intal, resumen Seminario Mercosur - Alianza del Pacífico 17 de junio de 2017. Recuperado de: https://conexionintal.iadb.org/2017/08/02/seminario-mercosur-alianza-del-pacifico/
} 
productivas y de valor más diversificadas y con mayor valor agregado que fortalezca la competitividad de la región. Desde los organismos internacionales y foros de discusión se señala los siguientes temas, identificados como puntos de intersección, donde coinciden los esfuerzos de los tres espacios de integración: acumulación de origen, facilitación de comercio, coherencia regulatoria, pymes, facilitación de comercio, inversión, innovación, ventanillas únicas de comercio y promoción comercial; sin dejar de lado, la agenda pendiente de integración física.

Por otro lado, en marzo de 2019, se dejó de lado a UNASUR, con la creación de PROSUR (Foro para el Progreso de América del Sur), implementada por Chile y Colombia, principalmente. Se ha dicho que UNASUR ha sido un instrumento chavista para impulsar el socialismo del siglo XXI. Sin embargo, es heredera de la Comunidad Sudamericana de Naciones, lanzada en el Cuzco en su momento. Esta fue propuesta por el Presidente Cardoso, como parte de la estrategia brasilera de consolidar no solo Mercosur, sino Sudamérica como parte de su proyección externa.

Esta política continuó con diferentes gobiernos. Era un proceso de integración con autonomía de la superpotencia, que buscaba una articulación con los BRICS, la cooperación sur-sur y la construcción de un mundo multipolar. Pese a sus limitaciones, permitió la coexistencia de procesos tan diversos como el ALBA y Alianza del Pacífico, consideramos errores de los primeros contribuyeron a precipitar la crisis. Se debería desideologizar el debate sobre la integración regional y su convergencia. El camino sería retomar las propuestas mencionadas anteriormente, y buscar acercamientos entre la CAN, Alianza del Pacífico y Mercosur, para avanzar por lo menos a nivel sudamericano. Serìa una convergencia bloque a bloque, ante la imposibilidad de hacerlo desde un organismo que incluya a todos (por la crisis de UNASUR que PROSUR no ha permitido superar).

En febrero de 2018, el Parlamento Andino, en el marco de su periodo ordinario de sesiones, aprobó la Recomendación $\mathrm{N}^{\circ}$ 345: "Para avanzar hacia la convergencia sudamericana de los procesos de integración CAN, UNASUR, MERCOSUR y la Alianza del Pacífico". Este instrumento propone a los países andinos avanzar en la convergencia y la diversidad, sumándose a las labores y esfuerzos que se vienen promoviendo en los diferentes foros de integración y sus puntos de intersección, siendo estratégicos para fortalecer la articulación productiva y la competitividad de nuestros países, así como la integración sudamericana.

Hoy más que nunca esa tarea está pendiente, ya que la reconfiguración del escenario mundial con la pandemia, dará más peso a la regionalización, en un mundo globalizado.

No hay condiciones para seguir con la misma estrategia de exportación de materias primas, y de inserción al inicio de las cadenas productivas globales. Se necesita construir nuevos motores de crecimiento, redefiniendo nuestro rol y participación en la economía mundial.

Podemos retomar una agenda de integración flexible, más allá de la coyuntura, buscando complementación productiva entre nuestros países, cadenas regionales de valor, esfuerzos de infraestructura conjuntos, facilitación de comercio, cooperación científica, servicios no tradicionales, industrias culturales, economía azul, energías renovables, bioeconomía, avanzando hacia una diversificación productiva que permita uso intensivo de capital humano y conocimientos que nos permitan una mejor inserción en la economía mundial (BID, 2020b; CEPAL, 2020a; CEPAL, 2020b). 


\section{Bibliografía}

Alianza del Pacífico (2018a) Alianza del Pacífico y Mercosur definen Plan de Acción para fortalecer vínculos entre los dos bloques. Recuperado de: https://alianzapacifico.net/alianzadel-pacifico-y-mercosur-definen-plan-de-accion-para-fortalecer-vinculos-entre-los-dosbloques/

Banco Interamericano de Desarrollo -BID (2018b). Estrategias y mecanismos para la convergencia de los acuerdos comerciales en América Latina. Recuperado de: https://publications.iadb.org/es/publicacion/14141/estrategias-y-mecanismos-para-laconvergencia-de-los-acuerdos-comerciales-en

Banco Interamericano de Desarrollo -BID (2020a) Cómo la política comercial puede ayudar a evitar una crisis alimentaria ante la pandemia de COVID-19. Sector de Integración y Comercio. Resumen de Políticas No IDB -PB- 337 Junio 2020. Recuperado de: https://publications.iadb.org/publications/spanish/document/Como-la-politica-comercialpuede-ayudar-a-evitar-una-crisis-alimentaria-ante-la-pandemia-de-COVID-19.pdf

Banco Interamericano de Desarrollo -BID (2020b) Informe macroeconómico de América Latina y el Caribe 2020: Políticas para combatir la pandemia. Recuperado de: https://flagships.iadb.org/es/MacroReport2020/Politicas-para-combatir-la-pandemia

Banco Mundial (2017). Mejores vecinos: Hacia una renovación de la integración económica en América Latina. Recuperado de: https://openknowledge.worldbank.org/bitstream/handle/10986/25736/210977ovSP.pdf

Comisión Económica para América Latina y el Caribe - CEPAL (2015) Panorama de la Inserción Internacional de América Latina y el Caribe 2015. La crisis del comercio regional: diagnóstico y perspectivas. Recuperado de: https://www.cepal.org/es/publicaciones/39010panorama-la-insercion-internacional-america-latina-caribe-2015-la-crisis

Comisión Económica para América Latina y el Caribe - CEPAL (2018a). Matriz de Insumo Producto Andina y las Cadenas de Valor. Recuperado de: https://www.cepal.org/sites/default/files/events/files/jose_duran__mip_y_cdv_comunidad_andina_0.pdf

Comisión Económica para América Latina y el Caribe - CEPAL (2018b). La convergencia entre la Alianza del Pacífico y el Mercosur. Recuperado de: https://repositorio.cepal.org/bitstream/handle/11362/43614/1/S1800528_es.pdf

Comisión Económica para América Latina y el Caribe-CEPAL (2020a) Informe sobre el impacto económico en América Latina y el Caribe de la enfermedad por coronavirus (COVID19). Recuperado de: https://www.cepal.org/es/publicaciones/45602-informe-impactoeconomico-america-latina-caribe-la-enfermedad-coronavirus-covid

Comisión Económica para América Latina y el Caribe - CEPAL (2020b) Dimensionar los efectos del COVID-19 para pensar en la reactivación. Informe Especial COVID 19 №2. 
Recuperado de: https://www.cepal.org/es/publicaciones/45445-dimensionar-efectos-covid-19pensar-la-reactivacion

Comisión Económica para América Latina y el Caribe -CEPAL-Organización de las Naciones Unidas para la Alimentación y la Agricultura - FAO (2020a) Análisis y respuestas de América Latina y el Caribe ante los efectos del COVID-19 en los sistemas alimentarios. Boletín $\mathrm{N}^{\circ} 1$. Recuperado de: https://www.cepal.org/es/publicaciones/45521-analisis-respuestas-americalatina-caribe-efectos-covid-19-sistemas-alimentarios

Comisión Económica para América Latina y el Caribe -CEPAL- Organización de las Naciones Unidas para la Alimentación y la Agricultura - FAO (2020b) Cómo evitar que la crisis del COVID-19 se transforme en una crisis alimentaria: Acciones urgentes contra el hambre en América Latina y el Caribe. Informe Covid CEPAL - FAO. Recuperado de: https://www.cepal.org/es/publicaciones/45702-como-evitar-que-la-crisis-covid-19-setransforme-crisis-alimentaria-acciones

CGTN (23/05/2019) El gobierno chino refuerza el apoyo al desarrollo de la red 5G. Recuperado de: https://www.youtube.com/watch?v=9rtMn5VTD_k

COSIPLAN (29/09/2005) Primera Reunión de Jefes de Estado de la Comunidad Sudamericana de Naciones. Recuperado de: http://cosiplan.org/Event/Detail?Id=146

Expansión (18/10/2017) Varios miembros de la OMC cuestionan la política proteccionista de Trump. Recuperado de:

https://expansion.mx/economia/2017/10/18/varios-miembros-de-la-omc-cuestionan-lapolitica-proteccionista-de-trump

Fairlie, Alan (24/05/2019) La guerra tecnológica entre China y Estados Unidos. Recuperado de: http://alanfairliereinoso.pe/?p=3811

Fairlie, Alan (5/10/2019) Guerra comercial entre China y Estados Unidos: entorno internacional preocupante. Recuperado de: http://alanfairliereinoso.pe/?p=4899

Fairlie, Alan (19/12/2019) Nueva negociación entre China y Estados Unidos. Recuperado de: http://alanfairliereinoso.pe/?p=5515

Fairlie Alan (26/12/2019) Ojalá se consolide el acuerdo inicial China y EE.UU. Recuperado de: http://alanfairliereinoso.pe/?p=5580

Fairlie, Alan (07/04/2020) Comercio de bienes esenciales en la pandemia. Recuperado de: http://alanfairliereinoso.pe/?p=6167

Fairlie, Alan (24/04/2020) Unilateralismo y multilateralismo en la pandemia. Recuperado de: http://alanfairliereinoso.pe/?p=6290

Gnangnon, S. (2019). Trade Policy Space, Economic Growth, and Transitional Convergence in terms of Economic Development. Journal of Economic Integration 2019, March;34(1) :1-37. Recuperado de: https://www.e-jei.org/upload/JEI_34_1_1_37_2013600179.pdf 
Japan Times (13/07/2017) TPP states agree new framework needed to implement pact without U.S. Recuperado de: http://www.bilaterals.org/?tpp-states-agree-new-framework\&lang=en

Kanitz, R. y Barros, M. (24/06/2017) Defensa comercial en la era Trump: proteccionismo y oportunidades para las exportaciones brasileñas. Recuperado de: https://www.ictsd.org/bridges-news/puentes/news/defensa-comercial-en-la-era-trumpproteccionismo-y-oportunidades-para-las

König, J. (2015). European Integration and the Effects of Country Size on Growth. Journal of Economic Integration, 30(3), 501-531. Recuperaco de: from www.jstor.org/stable/43549863 Organización Mundial de Comercio -OMC (8 de abril de 2020) Desplome del comercio ante la pandemia de COVID-19, que está perturbando la economía mundial. Comunicado de Prensa. Recuperado de: https://www.wto.org/spanish/news_s/pres20_s/pr855_s.htm

Peñaver, María (12/07/2017) El TPP se reúne en Japón para discutir su futuro. Recuperado de: http://www.bilaterals.org/?el-tpp-se-reune-en-japon-para\&lang=en

Secretaria General de la Comunidad Andina (22/10/2014) CAN y UNASUR trabajarán conjuntamente para promover convergencia de mecanismos regionales de integración Recuperado de: http://www.comunidadandina.org/Prensa. $\operatorname{aspx}$ ?id=3527\&accion=detalle\&cat=AF\&title=cany-unasur-trabajaran-conjuntamente-para-promover-convergencia-de-mecanismos-regionalesde-integracion

Sperlich, Y. y Sperlich, S. (2014). Income Inequality in the South-South Integration. Journal of Economic Integration 2014 December; 29(4): 726-758. Recuperado de: https://www.researchgate.net/publication/287305617_Income_Inequality_in_the_SouthSouth_Integration/citations

United Nations Conference on Trade and Development - UNCTAD (2020a) From the Great Lockdown to the Great Meltdown: Developing Country Debt in the Time of Covid-19. Trade and Development Report Update. Recuperado de: https://unctad.org/en/pages/PublicationWebflyer.aspx?publicationid=2710

United Nations Conference on Trade and Development - UNCTAD (2020b) The need to protect science, technology and innovation funding during and after the Covid-19 crisis. Policy brief No80. Recuperado https://unctad.org/en/pages/PublicationWebflyer.aspx?publicationid=2735

Xinhua Español (02/06/2019) China publica libro blanco sobre su postura en consultas económicas y comerciales con EEUU. Recuperado de: http://spanish.xinhuanet.com/201906/02/c_138110238.htm 